\title{
Mathematical and Ultrasonographic Model of the Left Ventricle: in Vitro Studies
}

\author{
Robert OLSZEWSKI ${ }^{(1)}$, Zbigniew TRAWIŃSKI ${ }^{(2)}$, Janusz WÓJCIK ${ }^{(2)}$, Andrzej NOWICKI ${ }^{(2)}$ \\ (1) Cardiology $\&$ Internal Medicine Clinic, Military Institute of Medicine, Cardiac Rapid Diagnostic Department \\ Szaserów 128, 04-141 Warszawa, Poland; e-mail: rolszewski@wim.mil.pl \\ (2) Institute of Fundamental Technological Research, Polish Academy of Sciences \\ Adolfa Pawinskiego 5B, 02-106 Warszawa, Poland \\ (received November 26, 2012; accepted December 5, 2012)
}

\begin{abstract}
The main objective of this study is to develop an echocardiographic model of the left ventricular and numerical modeling of the speckles- markers tracking in the ultrasound (ultrasonographic) imaging of the left ventricle. The work is aimed at the creation of controlled and mobile environment that enables to examine the relationships between left ventricular wall deformations and visualizations of these states in the form of echocardiographic imaging and relations between the dynamically changing distributions of tissue markers of studied structures.
\end{abstract}

Keywords: left ventricle, echocardiography, speckle modeling, ultrasound phantoms, strain, strain rate.

\section{Introduction}

Ultrasonography, an ultrasound-based diagnostic imaging technique, is currently the best available imaging technique to study human organs. Modern ultrasound equipment is constantly being upgraded with new functions and with more features based not only on the imaging or serving it, but also on parametric algorithms describing properties of tissues and objectifying diagnostics, which lead to the advanced research of the automatic remote systems of diagnostics

Two-dimensional echocardiography (2-D) constitutes this part of ultrasonography that is dedicated to heart imaging, which at present is the most frequently used imaging technique in the diagnostics of heart dysfunctions. This is so because 2-D echocardiography is noninvasive, relatively inexpensive and mobile technique, and thus readily available at the bedside; two-dimensional echocardiography allows that images are being created in the real time, the technique is available twenty-four hours a day, seven days per week which enables to run the accurate differential diagnosis in many health and life-threatening situations and/or for making correct diagnosis. Moreover it is subject to miniaturization and does not emit ionising radiation.

Risk of heart dysfunctions is increasing among growing part of population, most notably, its younger part (LLOYD-JonEs et al., 2010). It is a syndrome of modern-age diseases. Having inexpensive, reliable diagnostics, yet providing for the early and accurate detection of changes in the muscle structure would enable to lower the social costs of treating heart conditions and, what is more important, prevent many unexpected personal dramas.

Quantitative analysis of heart's functioning is the main weakness of two-dimensional echocardiography (2-D). Trials of applying the methods based on the Doppler signal such as tissue Doppler imaging (TDI) as well as strain and strain rate (SR) did not meet clinical expectations (ISAAZ, 2000; VAN DE VEIRE et al., 2008; Mor-Avi et al., 2011).

The new diagnostic method, speckle tracking echocardiography (2D STE) that is local scatterers tracking, which has recently been introduced in the clinical practice is very promising (LEITMAn et al., 2004; Korinek et al., 2005; Feigenbaum et al., 2005). However, this method should be free of errors that occurred when using previous methods. It is therefore essential to create the experimental numerical model of the left ventricle (based on equations of the theory of elasticity) and to establish the numerical model of ultrasound imaging process that would enable to conduct full analysis of the new method - 2D STE.

Problem with a quantitative analysis of the left ventricular (LV) function is a main weakness of twodimensional echocardiography (2D). Attempts to use 
methods based on the Doppler signal such as tissue Doppler imaging (TDI) on measurement of strain and strain rate $(S R)$ fell short of clinical expectations.

The first model - for the given boundary conditions - will enable to predict movement of the LV wall motion and wall material deformation and, in particular, scatterers displacements matching these deformations.

Second numerical visualization - for the propagation of the scanning beam, scattering of ultrasounds on tissue heterogeneity models in the moving wall will enable to detect echoes and create an image of the ventricular wall on the monitor (especially a speckle image and dynamics of this image during the movement of the ventricular wall).

A method, in order to be competitive in the field of echocardiography, must offer quantitative assessment of segmental impairments of left ventricular contractility. New 2D STE diagnostic method that has recently been introduced in the clinical practice should be an entirely understandable research tool. This is why it is necessary to create an experimental ultrasonographic model with a given wall material deformation, changing dynamics of the image during the movement of this wall, and especially velocity of the speckle tracking for various operating frequencies of the model.

In the last 50 years echocardiography has become clinical local and global method the most widely used in evaluation of the functioning of the left ventricle of the heart through the measurement of both the degree of change in the internal heart's size and left ventricular contractility. At first, only the distances between the selected points in 2D echocardiographic imaging were assessed. Next, the systems recording image and processing RF signals were used. There were great expectations in applying tissue Doppler imaging (TDI) technique, but this method did not give positive results in the assessment of heart wall (i.e., it has not adopted in its assessment the elasticity of the heart's wall).

The new technological solutions and concept of the tissue structures analysis, which were introduced in recent years, provide for more thorough, quantitative description of walls' deformation and blood flow in cardiac cavities. In the clinical practice, one of the most universal applications of the echocardiography is an assessment of the left ventricle ejection fraction (LVEF). Unfortunately, with currently used algorithms this task is partially subjective and time-consuming. Cases in which revealing pericardium (endocardium) outline is a difficult issue are real challenge to the method. Despite a great technological progress, in 10 to $20 \%$ of patients, especially among obese persons, it is impossible to obtain the optimal image (OLSZEWski et al., 2007). Another major limitation of echocardiography is the appropriate assessment of segmental contractility. Thus, quantitative methods to assess contractility impairments are necessary. For the last 20 years, attempts have been made to introduce a technique that would allow to analyze the operation of heart muscle both during the stress tests and during the rest. Unfortunately, subsequent methods that were introduced fell short of expectations.

One of the promising tools used it the last decade were the techniques examining the energies of the scattered echoes using unprocessed signal of ultrasound beam (integrated backscatter); however, this method was limited by lack of uniform processing and acquisition of data from the ultrasound beam (BIJNENs et al., 1999). TDI on the other hand, is limited by (like all Doppler techniques) the dependence of obtained value on the angle at which beam reflects from the moving cardiac muscle. Different parameters of TDI and deformation were studied in order to obtain quantitative values for the assessment of the left ventricular function, but these techniques did not produce the substantial progress.

Among the techniques used to study the elasticity of biological tissues, one deserves special attention. It is the method that consists in tracking the motion of acoustic tissue markers by analyzing the motion of socalled speckles in ultrasonographic imaging - in other words, speckles tracking. BOHS and TRAHEY (1991) who have developed a two-dimensional method of testing the displacement of the soft tissue with ultrasounds are considered to be precursors of this technique.

LANGELAND et al., (2003) were the first to use this type of pattern to assess the heart wall strains; they obtained linear correlation between the longitudinal and transverse strains and their designated estimators by using ultrasound technique, the method of RF signal correlation.

For about six years, clinical usage of the technique of speckles tracking has been researched. It has been widely publicized by the main producers of cardiologic (echocardiographic) equipment, even though none of the companies gives details of this technique. Moreover, the companies do not offer diagnostically significant mathematical expressions and terms of parameters used to describe heart's elastic properties. Lack of significant definitions of the assessed parameters leads to the inability to conduct comparative tests by using ultrasonographs made by different manufacturers. This problem has been noticed by the doctors conducting tests in large clinical centers, having at their disposal several devices made by different manufacturers. Unfortunately, people responsible for the promotion of these devices at the ultrasound conventions are unable to explain such a policy of huge concerns, and lack factual knowledge about the parametrization of elastic properties of heart wall.

\section{Physical bases of the speckle tracking}

Basic physical parameters of tissues, i.e., density, speed of sound and absorption parameter, are aver- 
aged for samples of the size of dozen or so to several dozens millimeters. In the homogeneous center values are equal to mean values. From microscale to millimeter scale, the existence of small deviations of the above mentioned parameters from the mean value is a natural quality of a tissue.

Depending on the type of variation, these inhomogeneities result from the tissue structure itself: from the combination with different weight of such qualities as fibrosity or granulation of stratification, from the mode of cell aggregation, from the interpenetration of different structures, such as the fibrous structure of the muscle tissue with the structure of small blood vessels and nerves. These inhomogeneities examined in the selected imaging plane constitute almost point microinclusions against a background of the homogeneous center. The resultant spatial distribution of these inhomogeneities has the random nature. It is usually described by the fixed in space probability density function of the occurrence of inhomogeneities.

Strain usually assigns every point of the solid to different point in space. From the mathematical viewpoint, it is a transformation that changes coordinates of the point masses of material region. These motions are accompanied by the velocity field of the region points. By rejecting translations and rotations of the region as a whole (with no additional dynamic effect), one obtains the result that agrees with the imaging notion of strain, even though in many cases strained body does not change its shape completely, or even its overall size. We are interested in the strains, in which there is a change of relative distances between points of the cardiac muscle fragments or the whole myocardium. Of great significance are especially these strains that lead to the change of size of the aforementioned areas, e.g., changes in the thickness of the ventricular wall.

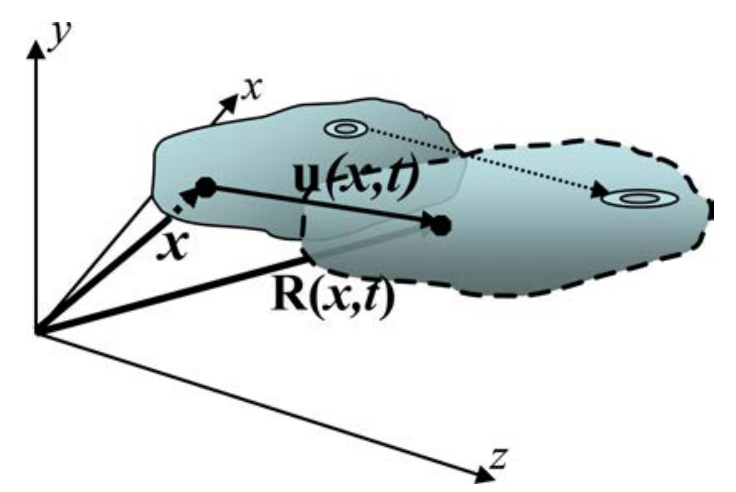

Fig. 1. An example of the deformation of tissue area and displacement of its points.

Possible results of the displacements of tissue are illustrated in Fig. 1. In this figure, we see displacement by vector $\mathbf{u}(\mathbf{x}, t)$ of the point mass of tissue with the initial position vector $\mathbf{x}$ to the position vector $\mathbf{R}(\mathbf{x}, t)=\mathbf{x}+\mathbf{u}(\mathbf{x}, t)$.
The general physical model describing the movement of the elastic medium is known (BLOTEKAER, SKEIE, 1973). It is described by the equation:

$$
g^{0} \partial_{u} \mathbf{u}=\nabla \circ \mathbf{P}, \quad g|J|=g^{0},
$$

where $\mathbf{u}=\mathbf{u}(\mathbf{x}, t), \mathbf{u} \equiv\left[u_{1}, u_{2}, u_{3}\right]$ is the displacement vector of the point with coordinate $\mathbf{x} \equiv\left[x_{1}, x_{2}, x_{3}\right]$ of the myocardium tissue with deformation $(\mathbf{x}, t) \rightarrow$ $(\mathbf{R}(\mathbf{x}, t)=\mathbf{x}+\mathbf{u}(\mathbf{x}, t), t)$; in Cartesian coordinates $\mathbf{x} \equiv[x, y, z],|J| \equiv|\partial \mathbf{R} / \partial \mathbf{x}|$ is the Jacobian of the transformation; $g=g(\mathbf{x}, t)$ is the density of the medium, though when in the initial state $t=t_{0}=0$, $\mathbf{u}\left(x, t=t_{0}\right)=\mathbf{0}$, then $g^{0}(\mathbf{x})=g\left(\mathbf{x}, t_{0}\right) ; \mathbf{P}=\mathbf{P}(\mathbf{u},\{\lambda\})$ is a stress tensor. In general, this tensor is non-linear because of $\mathbf{u}$ - the so-called geometric nonlinearity, independent of material properties; $\{\lambda\}$ is a set of material constants used to model physical properties of the medium; these are, for example, Lamé's constants or high-level constants, if there is the so-called physical nonlinearity; $\mid \nabla \circ P]_{m} \equiv \partial_{l} P_{m, l}$ is the tensor's divergence, where $P_{m, l} \equiv[\mathbf{P}]_{m, l} ; \nabla \equiv\left[\partial_{1}, \partial_{2}, \partial_{3}\right]$, $\partial_{l} \equiv \partial / \partial x_{l}$.

We assume that the medium is physically linear. Usually in the modeling the geometric linearity is also assumed, which comprises small displacements and small components of the relative deformation $\partial_{l} u_{m}$. The aforementioned assumptions for the homogeneous areas of the isotropic medium lead to the Lamé equation:

$$
g^{0} \partial_{u} \mathbf{u}=(\lambda+\mu) \nabla \nabla \cdot \mathbf{u}+\mu \Delta \mathbf{u},
$$

where $\lambda, \mu$ - Lamé's constants.

\section{Mathematical model of the left ventricle and acoustical channel}

Basic mathematical model was developed after observation of a human beating heart using a short axis parasternal view in $2 \mathrm{D}$ imaging. In this study, in order to develop numerical model of ultrasound imaging of the heart's walls (including the motion of speckles), the following assumptions were made:

1. From the geometrical viewpoint, the wall can be imitated by the cylindrical ring with an internal radius of $r 01$ and external of $r 02$ (diastolic) and the height larger than the width of the scanning beam.

2. In our basic numerical model ring's deformations are radially symmetrical. In every phase of contraction and diastole we have a ring with different radii of internal and external wall.

3. Microinhomogeneities are modeled with (random) distributions of point scatterers that at the same time function as ultrasound position (deformations) markers. 
To date, geometric nonlinearity has seldom been taken into consideration, while in fact we deal with the displacements of the order of the initial size of the modeled object's fragment; relative deformations can reach values of $0.5-1$.

Below we present an example of the numerical modeling of motion and deformation of the LV wall model. It has to be noted that the goal of the model is to provide the boundary conditions data on the real state of the movement of the wall. Calculations were made for the cylindrical ring of a shape and size of a segment of the real left ventricle. Similarly, boundary conditions (Fig. 2) were formulated on the basis of data from the real work of left ventricle.

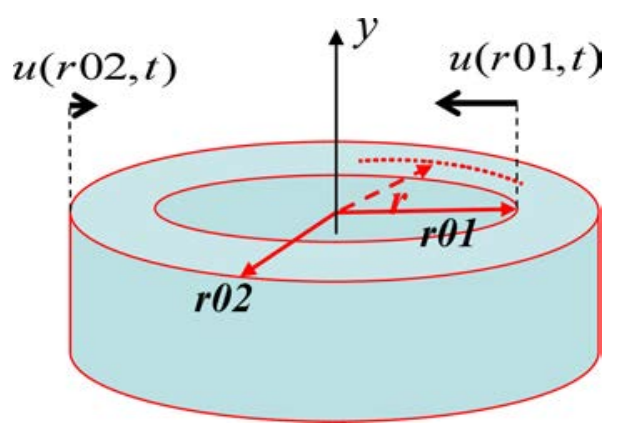

Fig. 2. Numerical model of the left ventricular wall (boundary conditions in diastole).

Cylindrical coordinate system was assumed. Axis y is a symmetry axis for the radially symmetrical ring deformation $R \equiv R_{r}(r, t)=r+u(r, t), \mid \mathbf{R}=R_{r}, R_{\varphi}=$ $0=R_{y}, u \equiv|\mathbf{u}|-u_{r}, u_{\varphi}=0=u_{y}, r 01 \leq r \leq r 02$ where $r 01=27.05 \mathrm{~mm}$ and $r 02=31.15 \mathrm{~mm}$ are the rings of the internal and external wall boundary in the initial state. We assumed diastole for the initial state $u(r, t=0)=0$. The following boundary conditions for the displacements were assumed: $u(r=r 01, t)=$ $0.5 d 1 \cdot(\cos (\omega t)-1), u(r=r 02, t)=0.5 d 2 \cdot(\cos (\omega t)-1)$, $d 1=6.84 \mathrm{~mm}, d 2=2.1 \mathrm{~mm}, \omega=2 \pi ; t=1$ is a conventional period of heartbeat: diastole: $t=0$; full contraction: $t=0.5$; diastole: $t=1$. Three radially symmetrical layers $0.8 \mathrm{~mm}$ thick were isolated in the wall, two layers by the boundaries and one inside.

Results are presented in the Cartesian coordinate system in the plane $z-x ; z$ - horizontal axis, $x-$ vertical axis. Wall boundaries in diastole are marked with red lines. Boundaries of the deformed wall for $t=0.125,0.25$ and 0.5 were marked with blue lines.

In order to model the acoustic channel and highlight the deformations in each of the layers within the wall area with an aperture of $-2.5^{\circ}$ and $2.5^{\circ}$ in the plane $z-x$, and $2 \mathrm{~mm}$ thick along the axis $y$, we randomly placed markers. Their initial positions were marked with red points. Blue points denote new positions of the markers (in the projection onto the plane $z-x)$ in the deformed layers for the aforementioned times (Fig. 3).

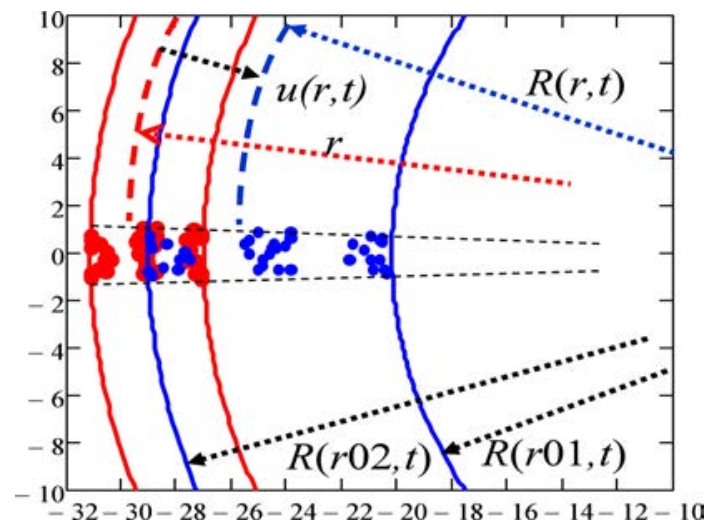

Fig. 3. General view of the deformed wall. Initial state (diastole) and positions of the tissue scatterers in three layers - in red. Final state (systole maximum contraction) and new positions of the markers - in blue.

a)

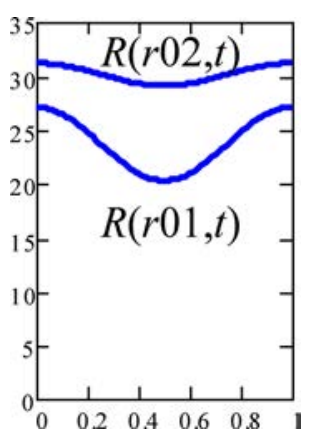

b)

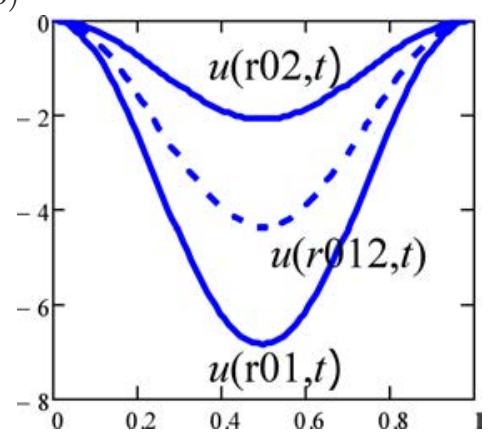

Fig. 4. a) The course of wall deformation during the whole cycle, b) the course of displacements of wall boundaries and middle internal layer $r=r 012=$ $(r 01+r 02) / 2$ (blue dotted line). Vertical axis scaled

in millimeters, horizontal axis - in time units.

The following processes form the basis of the ultrasonographic imaging:

- inducing ultrasonographic wave,

- dispersing or reflecting the wave in the tissue (creating echo),

- receiving echo and converting it into image.

When propagating through a homogeneous region, acoustic wave does not undergo scattering. Its scattering takes place in the inhomogeneous region. This indicates that inhomogeneities become the source of 
a) $t=0.125$

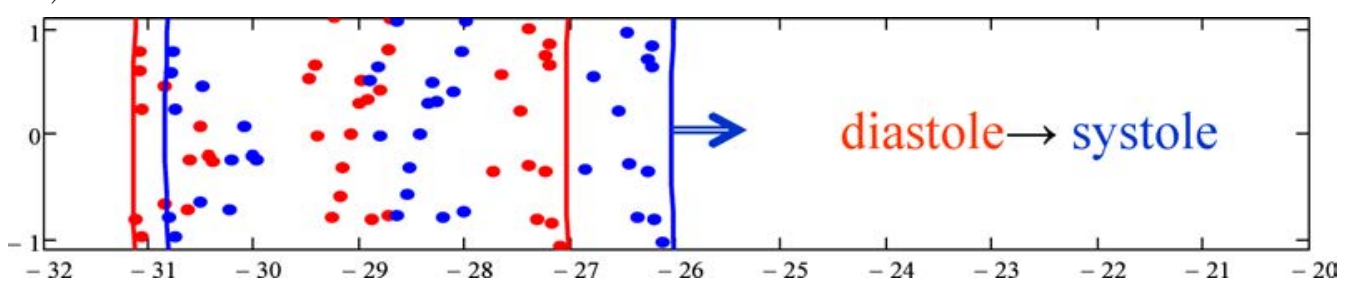

b) $t=0.25$

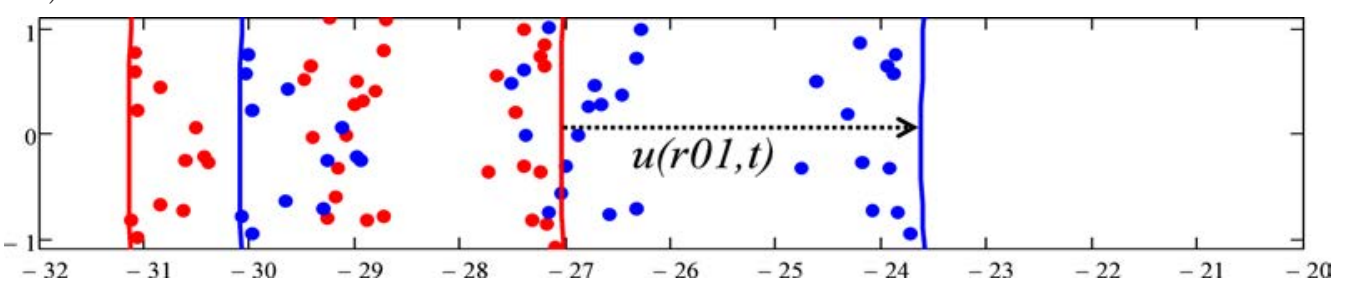

c) $t=0.5$

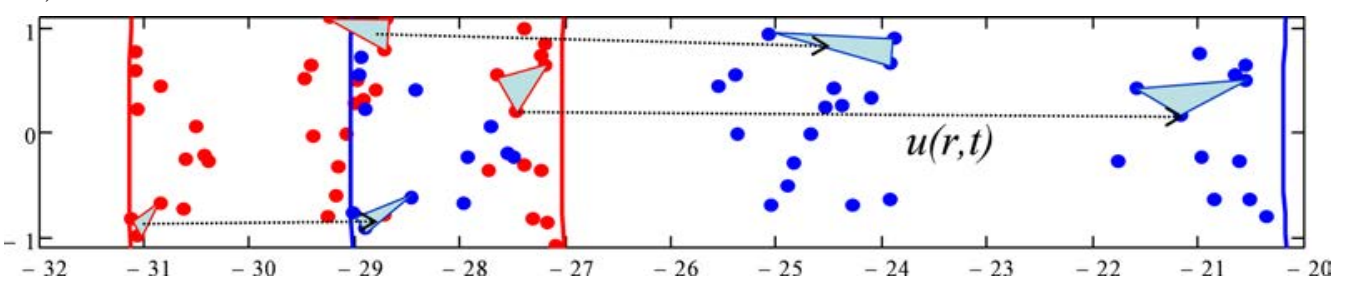

Fig. 5. Sequence of wall deformation illustrating scatterers displacement in each of the three layers depending on time: $t=0.125,0.25$ and 0.5 . In the diagram (c) we presented deformations of three areas (one in each layer) - each determined by three scatterers.

secondary waves. These waves interfere in space (i.e., their amplitudes are subtracting or adding themselves depending on the point in space). Interferential image is random if the distribution of dispersing regions is random.

Interference of the dispersed waves occurs also on the surface of a transmitter-receiver head that induces electric signal, larger - when waves' amplitudes are adding themselves on its surface, and smaller - when they are subtracting themselves. This signal controls larger or smaller brightening of screen pixels along the line which is an axis of the scanning beam (Fig. 6). Change in the position of dispersing regions in time causes change in the position of pixels' brightenings along the image line.

Pathological changes occurring in the cardiac muscle change both size and spatial distribution of these inhomogeneities of physical parameters.

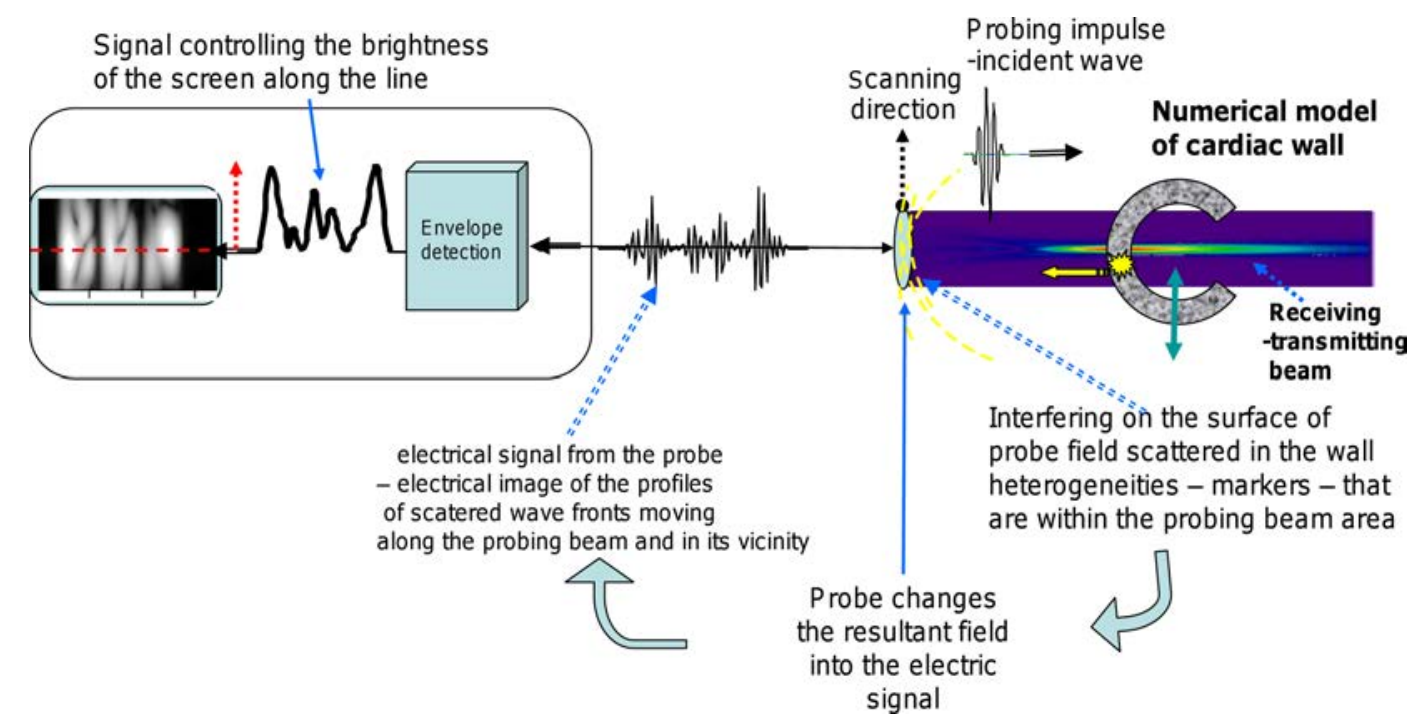

Fig. 6. Acoustic track diagram. 
Physical model describing the phenomenon of acoustic field generation, its propagation and scattering in the inhomogenous region and its detection, is well-known. Basic equation of the model is SturmLiouville's equation (modified Helmholtz' equation) (WóJCIK et al., 2006; 2008). Integral form of this equation is

$$
\begin{aligned}
C\left(\mathbf{R}^{\prime}, n\right)= & C^{t}\left(\mathbf{R}^{\prime}, n\right)-n^{2} \int_{v} G\left(\mathbf{R}^{\prime}-\mathbf{R}^{\prime \prime}, n\right) \\
& \cdot V\left(\mathbf{R}^{\prime \prime}\right) C\left(\mathbf{R}^{\prime \prime}, n\right) \mathrm{d} v,
\end{aligned}
$$

where $C^{t}\left(\mathbf{R}^{\prime}, n\right)$ - spatial distribution of $n$-th component of Fourier's incident wave, which is a solution of the homogenous Helmholtz equation, $C\left(\mathbf{R}^{\prime}, n\right)-$ total area (incident and dispersed) in space, $\mathbf{R}^{\prime} \equiv[z, \mathbf{r}]^{\prime}$ - coordinate vector is space, $\mathbf{R}^{\prime \prime} \equiv[z, \mathbf{r}]^{\prime \prime}$ coordinate vector in area $v$ taken by inhomogeneities of the tissue described with dispersing potential $V, \mathbf{r} \equiv[x, y]$. $G(\mathbf{R}, n)=G(R, n) \equiv \exp (i \cdot n \cdot R) / 4 \pi \cdot R$ is Green's function of the Helmholtz equation, $R \equiv|\mathbf{R}|$.

Let us assume for the moment that scattering occurs on one scatterer, point inhomogeneity with a task force of $\nu$ that can take any coordinate $\mathbf{R} \equiv[z, \mathbf{r}]$ in the area of imaging. Then, in Eq. (1) we write

$$
\begin{aligned}
V\left(\mathbf{R}^{\prime \prime}\right) & \equiv \nu \delta\left(\mathbf{R}^{\prime \prime}-\mathbf{R}\right), \\
\nu & =\text { const }
\end{aligned}
$$

where $\delta(\cdot)$ is the Dirac distribution. The above assumption is sufficient to properly analyze the ultrasonographic track, that is transmitting and receiving features of transducers and their systems, and basic phenomena connected with tissue imaging.

Dispersed field $C^{r} \equiv C-C^{t}$ with the disclosed task $\mathbf{R}$ coordinate, determined on the basis of Eqs. (1) and (2), is given by the formula

$$
C^{r}\left(\mathbf{R}^{\prime}, \mathbf{R}, n\right)=-n^{2} \nu G\left(\mathbf{R}^{\prime}-\mathbf{R}, n\right) C^{t}(\mathbf{R}, n) .
$$

Electric signal (here, its Fourier's spectrum), induced by the scatering fields incidenting on the surfaces $\Pi$ of the transducer and registered in the receiving track, is described with the formula

$$
\begin{aligned}
E(\mathbf{R}, n) & =c^{r}(n) \cdot \int_{\Pi} C^{r}\left(\mathbf{R}^{\prime}, \mathbf{R}, n\right) \mathrm{d} \Pi \\
& =n^{2} c^{r}(n) \nu g^{r}(\mathbf{R}, n) c^{t}(n) g^{t}(\mathbf{R}, n),
\end{aligned}
$$

where $E(\mathbf{R}, t)=F^{-1}[E(\mathbf{R}, n)] ; c^{r}(n)$ is a frequential receiving feature, cumulating electromechanical and electrical properties of the transducer and registration channel; $c^{t}(n)$ is spectrum (phantom) of the transmitted impulse, $C^{t}(\mathbf{R}, n)=c^{t}(n) g^{t}(\mathbf{R}, n)$. Characteristics of transmitting $g^{t}$ and receiving $g^{r}$ constitute the solutions of the Helmholtz equation for boundary problem $z=0$ of Dirichlet and Neumann for half-space $z \geq 0$, respectively. They are given by the formulas:

$$
\begin{aligned}
& g^{r}(\mathbf{R}, n)=\Pi(\mathbf{r}) \underset{\mathbf{r}}{\otimes} G(\mathbf{R}, n), \\
& g^{t}(\mathbf{R}, n)=\Pi(\mathbf{r}) \underset{\mathbf{r}}{\otimes} G^{z}(\mathbf{R}, n), \\
& G^{z}(R, n) \equiv 2 \partial_{z} G(R, n),
\end{aligned}
$$

where $G^{z}$ is the Green function for a Dirichlet problem. Functions (e) $\Pi(\mathbf{r})$ are characteristic functions of the transducer, describing its load (apodizations). We assume that the transmitting transducer is also a receiving one (as it is usually in heart diagnostics) and it is loaded evenly when transmitting and receiving, that is that is $\Pi(\mathbf{r})=1$ for $\mathbf{r}$ corresponding to active while transmitting or sensitive in reception transducers points and $\Pi(\mathbf{r})=0$ for the remaining $\mathbf{r}$.

Spatial formulas of the transmitting and receiving characteristics based on Eqs. (4) and (5) can be written down as

$$
\Gamma(\mathbf{R}, n) \equiv g^{t}(\mathbf{R}, n) g^{r}(\mathbf{R}, n)=\partial_{z}\left(g^{r}(\mathbf{R}, n)\right)^{2} .
$$

Electromechanical properties of the electronical track are described with the formula $c^{r t}(n) \equiv$ $c^{r}(n) c^{t}(n)$. Thus, Fourier's spectrum (4) of the signal received from the scatterer in the point of $\mathbf{R}$ has the following form:

$$
E(\mathbf{R}, n)=n^{2} c^{r t}(n) \cdot \nu \cdot \Gamma(\mathbf{R}, n) .
$$

An example of the spatial distribution of $|\Gamma(z, r, n)|$ of an axisymmetrical beam generated by the transducer with a diameter of $15 \mathrm{~mm}$ for $n=3.5 \mathrm{MHz}$, corresponding to the carrier frequency of the probing beam, is presented in Fig. 7; $z$ - beam axis; $r=|\mathbf{r}|=\sqrt{x^{2}+y^{2}}-$ lateral coordinate. Axes are scaled in millimeters.

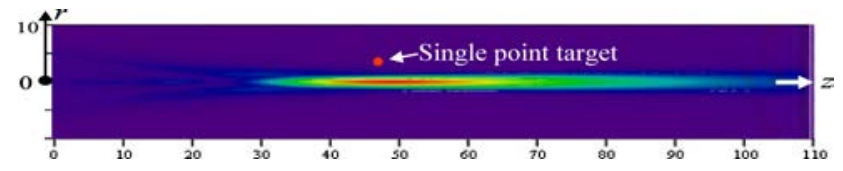

Fig. 7. Transmitting and receiving characteristics of the transducer for $n$ frequency corresponding to $3.5 \mathrm{MHz}$.

Angular scanning consists in turning the transducer towards the given axis (here $y$ ). In order to avoid unnecessary complications of the record connected with the introduction of turn transformation to the description of the angular scanning (that do not contribute to the understanding of the principles of operation of the acoustic track and creation of the image), next we consider only the linear scanning.

With the translation by vector $\mathbf{r}_{s}$, in a fixed coordinate system, function $E(\mathbf{R}, n)$ (signal's spectrum) becomes the equation

$$
\begin{aligned}
E^{s}\left(n, \mathbf{r}_{s} ; \mathbf{R}\right) & \equiv E\left(\mathbf{R}-\mathbf{r}_{s}, n\right) \\
& =n^{2} c^{r t}(n) \cdot \nu \cdot \Gamma\left(\mathbf{R}-\mathbf{r}_{s}, n\right) .
\end{aligned}
$$


If $\mathbf{R}=\mathbf{R}_{1}$ is a set position of the real point scatterer (Fig. 10) and the range $\mathbf{r}_{s}$ of scanning is wide enough, so that beam "cuts" the object during scanning with its whole width, then the spectrum of the registered signal has the form of $E^{s}\left(n, \mathbf{r}_{s} ; \mathbf{R}\right) \equiv E\left(\mathbf{R}_{1}-\mathbf{r}_{s}, n\right)$. We obtain the combined spatial image by calculating and plotting in the coordinates functions $\left(z, \mathbf{r}_{s}=[x, 0]\right)$.

$$
P^{s}\left(z, \mathbf{r}_{s} ; \mathbf{R}_{1}\right) \equiv F^{-1}\left\lfloor E^{s}\left(n, \mathbf{r}_{s} ; \mathbf{R}_{1}\right)\right\rfloor .
$$

Localization and size of the target in $z$ coordinate result from the dependence $z \equiv c_{0} t / 2$ (in the assumed system of units $c_{0}=1$ ). With the boundary detection on the ultrasound scanner screen, the image of values $\left|P^{s}\left(z, \mathbf{r}_{s} ; \mathbf{R}_{1}\right)\right|$ forms, usually in grey scale. It is the socalled point spread function (PSF) - image of the point object obtained as a result of scanning with ultrasound beam. It is obvious that the form and the size of PSF depend on the length of the impulse (along $z$ ) and transversal size of the beam on the plane $z=$ const. $(x-y)$ in which there is the target - point marker.

PSF image with unit task force of $\nu=1$ can be considered the image of the elementary speckle. If we neglect the echoes from lateral bands of the scanning beam this is always a single speck. Its shape and size can change (to a small extent in case of well-constructed scanning beams) while the scatterer moves, as it depends on its position relative to the scanning beam.

In the tissue there are many scatterers (about 30 $\mathrm{l} / \mathrm{mm}^{3}$ ) with random distribution of positions $\left\{\mathbf{R}_{l}\right\}$ and target forces $\left\{v_{l}\right\}, l=1, \ldots, L$. Equation (2) takes the form:

$$
V\left(\mathbf{R}^{\prime \prime}\right) \equiv \sum_{l}^{L} \nu_{l} \delta\left(\mathbf{R}^{\prime \prime}-\mathbf{R}_{l}\right)
$$

The signal's spectrum is now a sum of elementary spectra (10) and the emerging image is a result of the interference of many combined images of the elementary speck-speckles, like in Figs. 8 and 9. With boundary detection the distribution of energy in the image is given by the formula

$$
\begin{aligned}
\left|P^{s}\left(z, \mathbf{r}_{s}\right)\right|^{2} & =\sum_{l}\left|P^{s}\left(z, \mathbf{R}_{l}-\mathbf{r}_{s}\right)\right|^{2} \\
& +2 \sum_{\substack{l, m \\
l \neq m}} \operatorname{Re}\left(P^{s}\left(z, \mathbf{R}_{l}-\mathbf{r}_{s}\right) P^{s}\left(z, \mathbf{R}_{m}-\mathbf{r}_{s}\right)^{*}\right) .
\end{aligned}
$$

Even though there is a reciprocal equivalence between scatterers distribution (12) and their image (13), only in case of properly, sparsely distributed markers the interference segment - second on the right in Eq. (13) - can be omitted and positions of the scatterers can be read off directly from the position of speckles on the screen. Such a situation can be accomplished by the use of numerical or laboratory model. a)

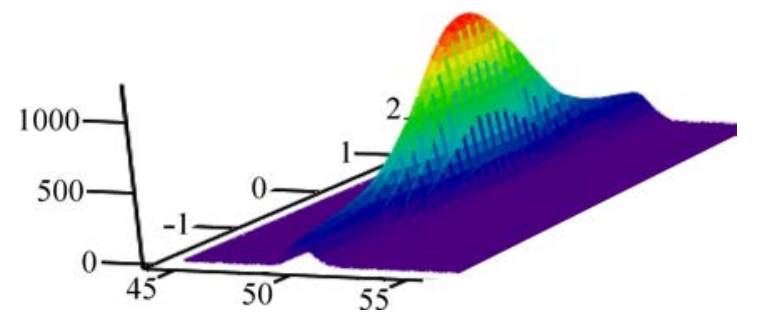

b)

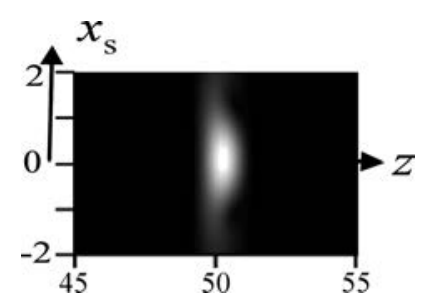

Fig. 8. a) perspective view of the value of profile PSF, b) PSF images in shades of gray with the boundary detection for the beam described in Fig. 7 and the scatterer on the plane $z=50 \mathrm{~mm}$.

a)

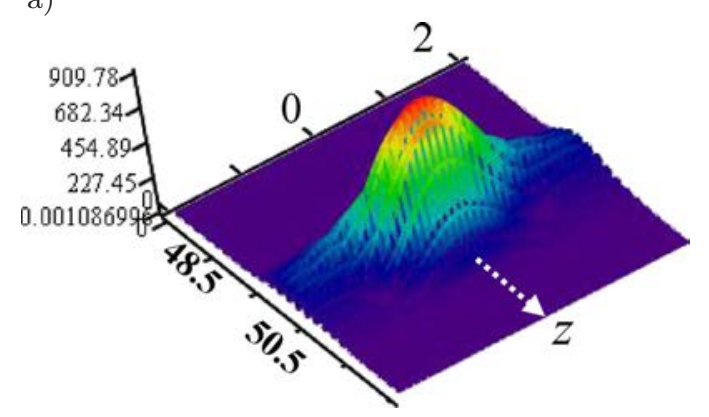

b)

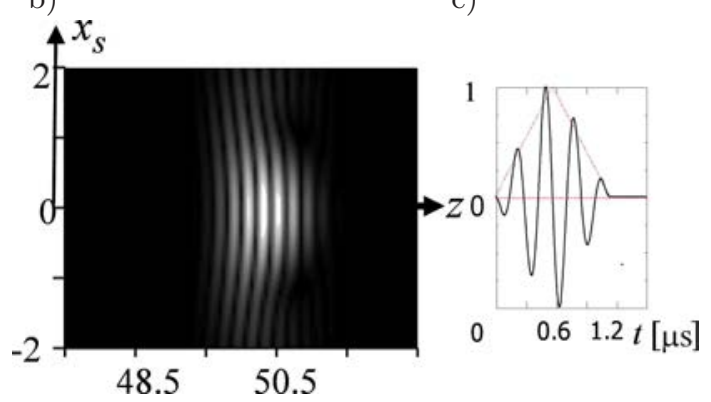

Fig. 9. PSF details: a, b) images of the absolute value of the real part of PSF, c) normalized timing of the transmitted scanning impulse.

It should be noted that the above formulas are correct for the static object with the distributing potential $V(\cdot)$ that is not depending on time $t$. In our case, markers' position, by default, depend on time $\mathbf{R}_{l}=\mathbf{R}_{l}(t)$. However, if characteristic time of changes (deformations) of the object $\tau_{d}$ is much larger than doubled time $\tau_{c}$, in which the probing impulse "cuts" the object along scanning line, then the object can be regarded as quasi-static. In our case the estimated doubled timing of impulse going through the wall in the contraction 
is $2 \tau_{c} \propto 2 \cdot 10^{-2}[\mathrm{~m}] / 1500[\mathrm{~m} / \mathrm{s}] \approx 13 \cdot 10^{-6}[\mathrm{~s}]$, while the period of heartbeat is $\tau_{d} \approx 1 \mathrm{~s}$. Finally, the ratio of maximum deformation speed to the speed of sound is $x / c_{0}<3 \cdot 10^{-5} \ll 1$. Thus, the ventricular wall can be regarded as static object in scales of time 100-1000 times higher, complying with time in which the modern ultrasound scanners need to create multilinear $2 \mathrm{D}$ image.

Thus, we can rewrite Eq. (13), revealing through $\mathbf{R}_{l}(t)$ the dependence on time:

$$
\begin{aligned}
& \left|P^{s}\left(z, \mathbf{r}_{s} ; t\right)\right|^{2}=\sum_{l}\left|P^{s}\left(z, \mathbf{R}_{l}(t)-\mathbf{r}_{s}\right)\right|^{2} \\
& \quad+2 \sum_{\substack{l, m \\
l \neq m}} \operatorname{Re}\left(P^{s}\left(z, \mathbf{R}_{l}-\mathbf{r}_{s}\right) P^{s}\left(z, \mathbf{R}_{m}(t)-\mathbf{r}_{s}\right)^{*}\right) .
\end{aligned}
$$

It can be demonstrated that Eq. (14) will remain in its overall form even after the more detailed analysis conducted only in the field of space-time (it includes effects omitted in case of the assumption about quasistatic state. One can be surprised at the introduction of time dependence into the Fourier spectrum. However, we must remember that time "numbers, parametrizes" here states (stages of heartbeat) and a spectrum is the spectrum of impulse and signals dispersed over the "frozen" heart in a time period of $\left[t-\tau_{c} ; t+\tau_{c}\right]$ (or even much longer) and in the area of beam probing the heart wall. Below we present images $\left|P^{s}\left(z, \mathbf{r}_{s} ; t\right)\right|$ obtained as a result of numerical modeling of the process of visualization of scatterers distribution for the presented above numerical model of the left ventricle for three different moments in time and two different concentrations. General field of displacements is the same as in Figs. 3, 5. Scanning schema is presented in Fig. 10. Probing range $r_{s}=\left[x_{s}, 0\right]$ was selected in such a way that the scanning beam "cuts| with all its effective width all of the scatterers in the imaging field. Figure 11 corresponds to the aforementioned situation of sparsely (one per layer, $\sim 0.25 \mathrm{l} / \mathrm{mm}^{3}$ ) distributed scatterers - there are no interference expressions in Eqs. (13) and (14). Figure 12 is a sequence of three images that precisely matches the sequence of three stages of deformation and corresponding to them distributions of the scatterers presented in Fig. 5.

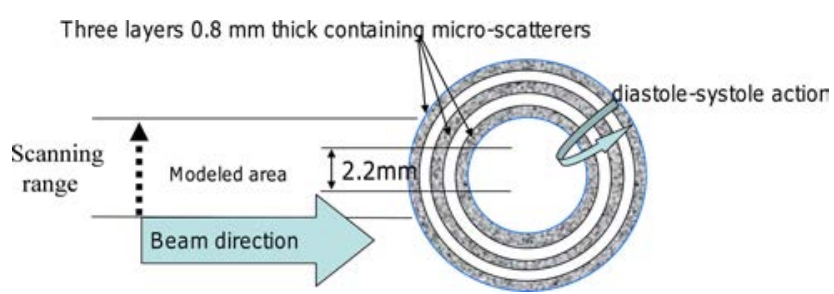

Fig. 10. A diagram of scanning.

The size of the speckle to a small extent is connected with the local distribution of sources (i.e., small inhomogeneities, differences in the density understood

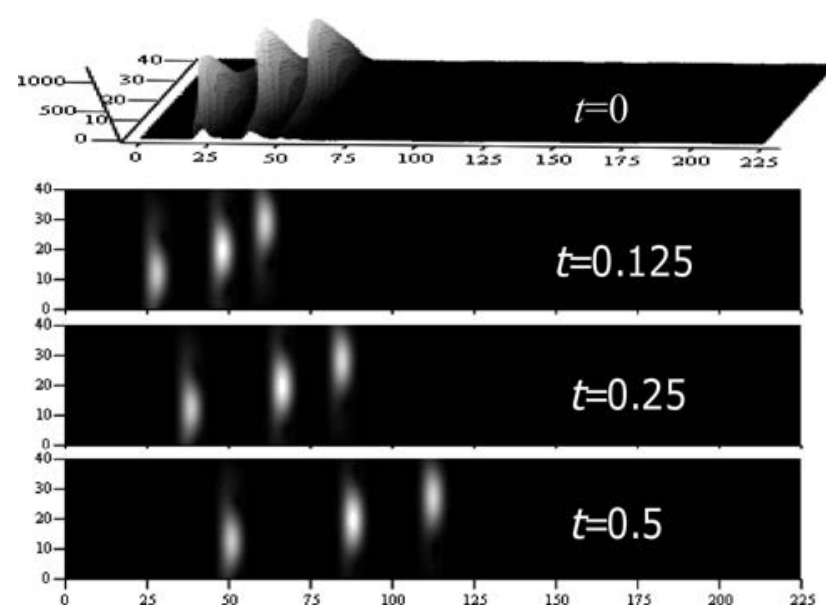

Fig. 11. Distribution of the image of scatterers. Loose distribution, one per each layer. Result - image as a simple sum of PSF. Axis unit is $0.1 \mathrm{~mm}$ - scanning step towards the vertical axis $x_{s}$.

\section{Scanning direction}

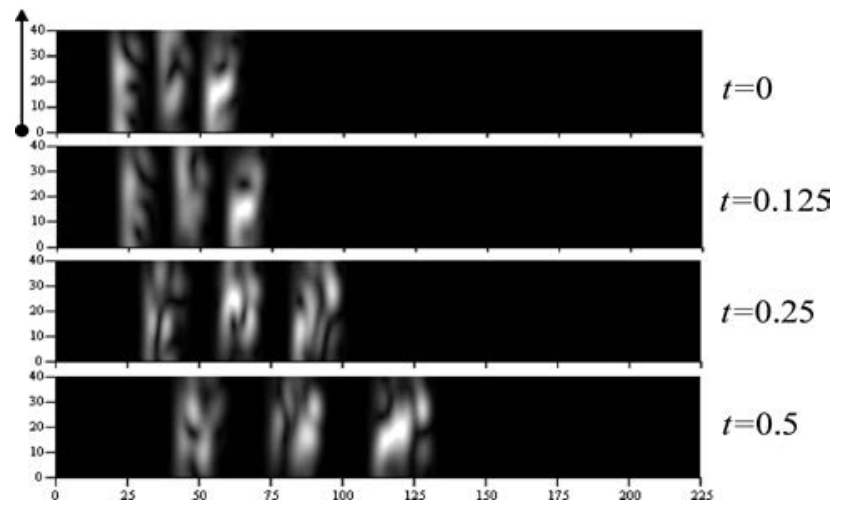

Fig. 12. Distribution of the image of scatterers. Distribution of the markers - as in Fig. 5. Distribution of the markers, respectively: $12-16-12,\left(\sim 3-4 \mathrm{l} / \mathrm{mm}^{3}\right)$. Result - complex image of type (13). Units are as in Fig. 11.

as minor when compared with the wavelength) scattering the wave. The size of the speckle is more dependent on the distribution of the ultrasound field. Working on the generally accepted assumption that scattering is a random process with the Rayleigh distribution that acts like multiplicative noise, the following were determined: the average size of speckle, thickness of speckle (in the radial axis $\mathrm{x}$ ), and width of the speckle (on the vertical plane from the radial axis) for the impulses with envelope in the form of the Gauss curve with the standard deviation $\sigma$.

$$
\begin{aligned}
\text { speckle }_{x}= & 2.43 \cdot \sigma=1.02(\text { impulse Full Length } \\
& \text { at Half Maximum FLHM), }
\end{aligned}
$$

speckle $_{y, z}=0.94 \frac{\lambda F}{2 a}$.

Speckle size is approximately comparable to the spatial size of the probing impulse (WAGNER et al., 1983). Ultrasonographs with incorrectly focused beams and long 
"ringing" impulses have large speckle and, because of that, low contrast resolution.

\section{An example of using the calculated algorithms}

Both models were integrated, especially in the context of the description of the deformation of LV wall - including various (also random) distributions of the distribution of local changes in the density of tissue that is characterized by the distinctive granularity (speckles).

Thus, it can be concluded that small areas of the cardiac muscle, with their unique point model, can be defined as the pattern for the group of speckles. Image of this model in the cardiac muscle can be observed with commercially available software during the cardiac cycle. In the technique of speckles tracking a particular region of interest (ROI)) is defined, which can be observed by applying the relevant algorithm. The algorithm examines the most similar distributions of speckles while going through the adjacent, previously recorded image cells during the cardiac cycle For example, the algorithm may examine areas with the smallest difference in the total number of pixels (bright) or use the correlation methods. The described technique does not depend on the angle of incidence of the ultrasonic beam, since

a)

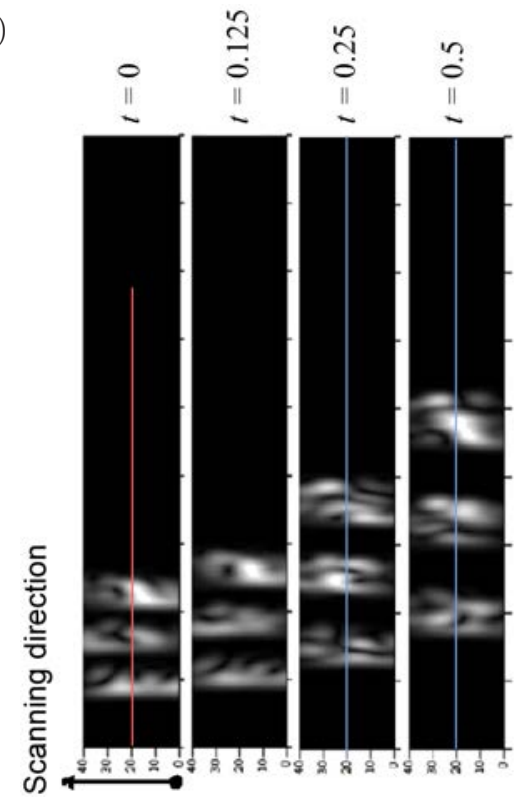

b)

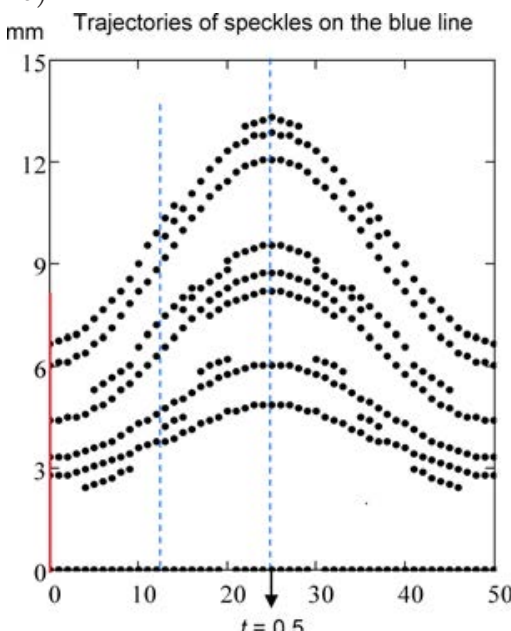

b)

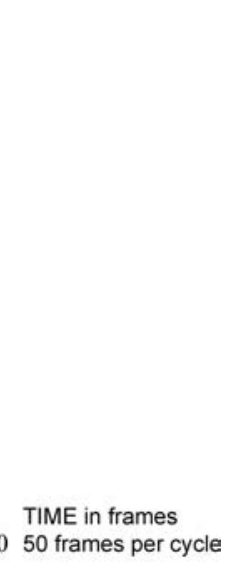

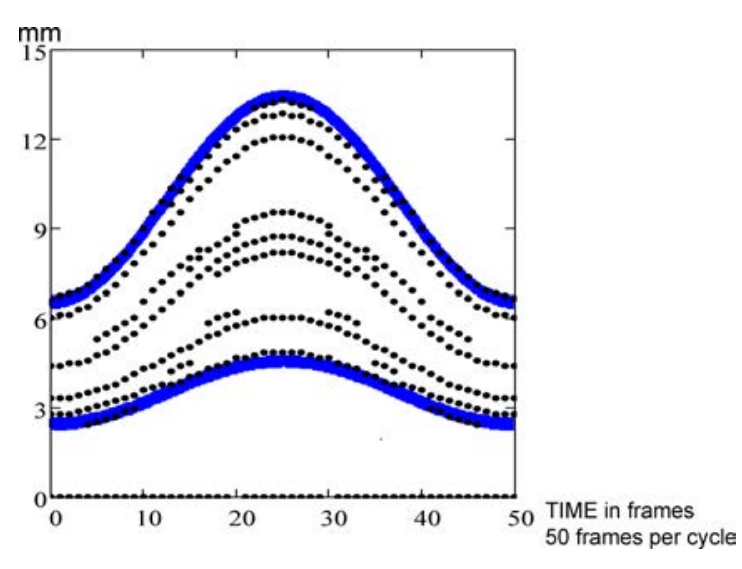

Fig. 13. The result of modeling, motion detection and following the movement of acoustic markers: a) image of speckles for four phases of contraction of the model of ventricle (from diastole $t=0$ do full contraction $t=0.5$ ), b) detected on the basis of the analysis of the speckles image (a) - along blue line on (a) - position of the real and apparent markers (scatterers) in three layers during the whole cardiac cycle. Trajectories of the markers determined on the basis of the analysis of the motion of real and interference speckles, c) same as (b) with real positions (thick blue lines) of the boundaries of ventricle model (trajectories of speckles on the blue line; TIME in frames 50 frames per cycle). 
it is based on the displacement of speckles, the distribution of which is connected with characteristic features of cardiac muscle walls, rather than with characteristic features of the ultrasonic beam (with set beam values) as it is in Doppler's technique. It should be point out that, in the technology of speckles tracking, the image is already sufficiently complex and with the concentration of scatterers about ten times smaller than average real tissue, the image is complex enough and varies significantly in the course of deformation. This sets very high requirements for the aforementioned tracking algorithms.

The percentage change of sizes (length, thickness) of examined object (compared with its previous size) is called Lagrangian strain in diagnostics. Strain and strain rate (SR) imaging enable to take segmental measurement of the myocardium strain for the assessment of its local and global functioning. Strain measures the relative strain (if left ventricle wall size increases by one-fourth, then strain amounts to $25 \%$ and if it decreases by three-fourths then the strain amounts to $-75 \%$ ). Strain rate represents the changes in strain per time unit. Both parameters provide information about heart functions that complement each other. Strain and $S R$ values obtained in the echocardiography were verified in vitro and in vivo by using various methods. One of the simple ways of assessing strain is by using the parasternal short axis M-mode view and calculating it from the thickness of wall during the contraction (systole).

The model of the left ventricle we propose is the realization of a fully controlled diagnostic environment, allowing for the testing of algorithms that follow and analyze dispersion of acoustic markers, as well as improving the existing algorithms or supporting the construction of new ones. Based on the mathematical model, the ultrasonographic model of left ventricle was built.

Model of the left ventricle (LV) was fabricated from POLY (VINYL ALCOHOL), 99+\% HYDROLYZED material and had a shape of a tube with the internal diameter of $5 \mathrm{~cm}$, wall thickness of $1 \mathrm{~cm}$ and length of $12 \mathrm{~cm}$. Acoustic properties of the phantom material were close to that of the real tissue: speed of the ultrasounds $=1546 \mathrm{~m} / \mathrm{s}$, absorption coefficient $=0.28 \mathrm{~dB} / \mathrm{cm}($ at $2.95 \mathrm{MHz}), \mathrm{SNR}=10.8 \mathrm{~dB}$ (at $3.5 \mathrm{MHz}$ ). Figure 14a shows the image (view) of polyvinyl alcohol material sample, structure under an optic microscope, and an echogram of this sample, respectively (Fig. 14).

The LV phantom was driven using the automatic and computer-controlled hydraulic piston pump (Vivitro Inc., Canada) with stroke volume in the 10-100 $\mathrm{ml}$ range. It pumps fluid at pressure of $0-300 \mathrm{mmHg}$, at the frequency of 30-120 impactions per minute for 100-700 ms, allowing for the performance of pulsatile filling of the model of the left ventricle with fluid with a)

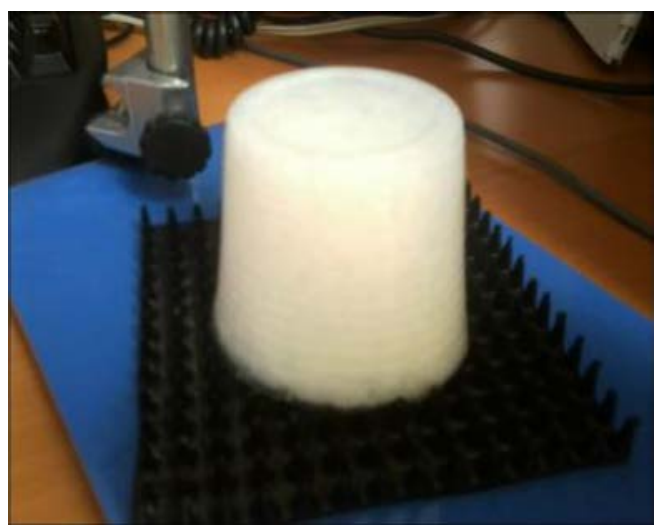

b)

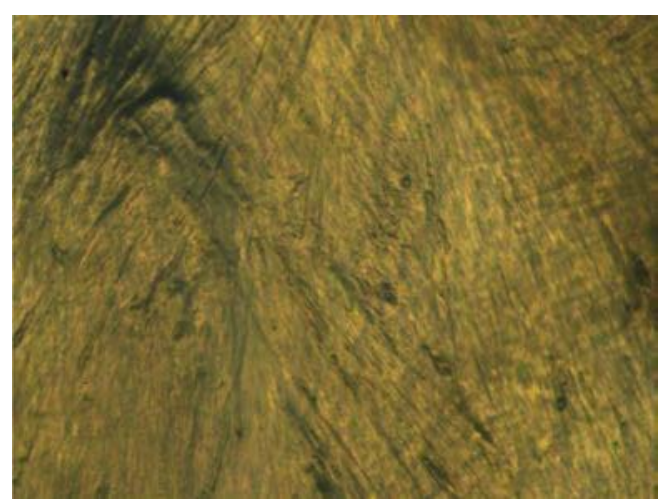

c)

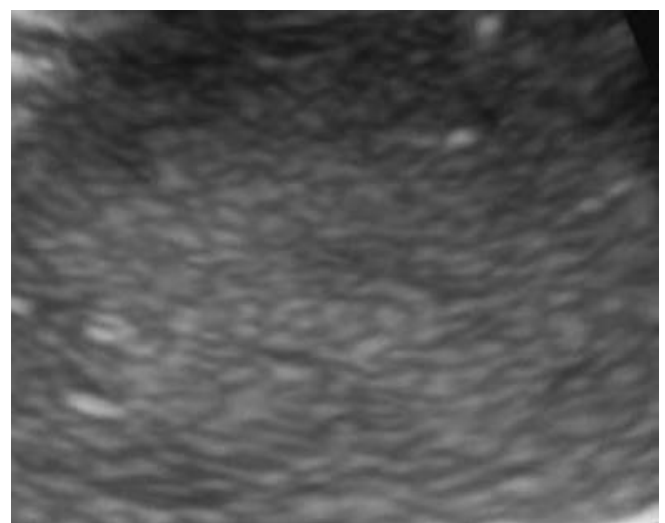

Fig. 14. Polyvinyl alcohol used to build the model of left ventricle (LV): a) image of the sample from the model of left ventricle built from (made of) the polyvinyl alcohol, b) image of the sample under light microscope, c) ultrasound image of the sample with visible speckles - acoustic markers.

precisely defined filling level. The phantom displacement was examined using: Artida 4D echocardiograph (Toshiba Medical Systems, Tokyo, Japan) and Acuson X 300 (Siemens Medical System, USA). Figure 15 is a block diagram of the experimental set-up. Models of the left ventricle were examined with commercially available 2D STE algorithms using the following parameters: pumping water volumes of 12-, 24-, 36-, 40-, $50 \mathrm{ml}$ with frequency of 40,60, 100 and 120 cycles per minute, for ultrasound beam angles equal to $90^{\circ}$ and $65^{\circ}$ towards axis of the model. 


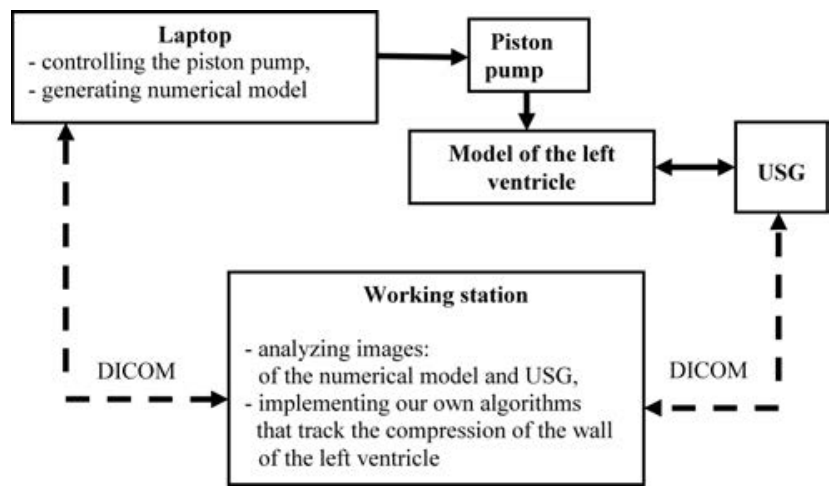

Fig. 15. Block diagram of the echocardiographic model of the left ventricle.

2D STE imaging of the LV phantom, for small and large amount of pumped out water, is presented in Fig. 16 and 17. Strain values of this model for the volume of given water in one projection for volume of 12 , 24 and $36 \mathrm{ml}$ and the frequency of 40,60, 100 and 120 cycles per minute for each of the samples were obtained automatically during all measurements in $100 \%$.

a)

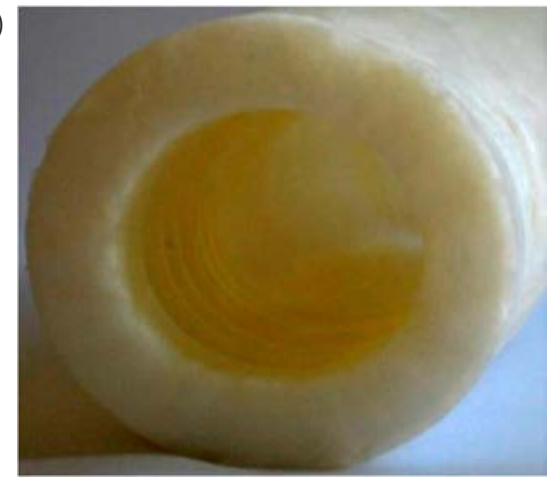

b)

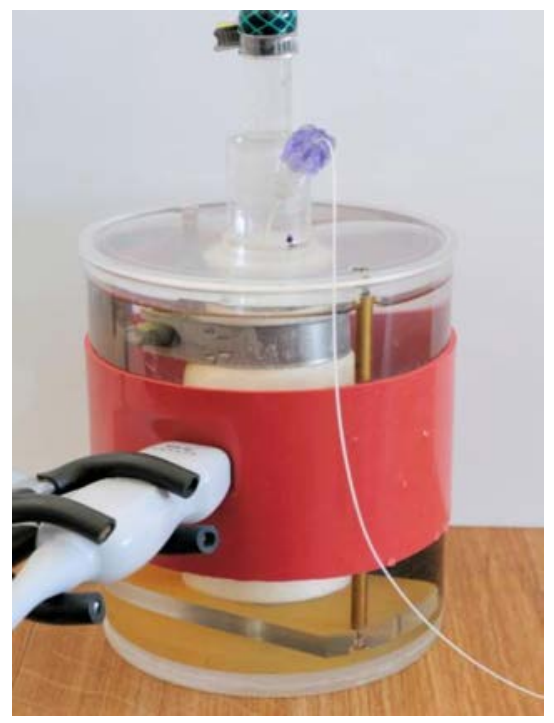

Fig. 16. a) image of the cross-section of the left ventricle model, b) photo of the left ventricle echocardiographic model during the image acquisition. a)

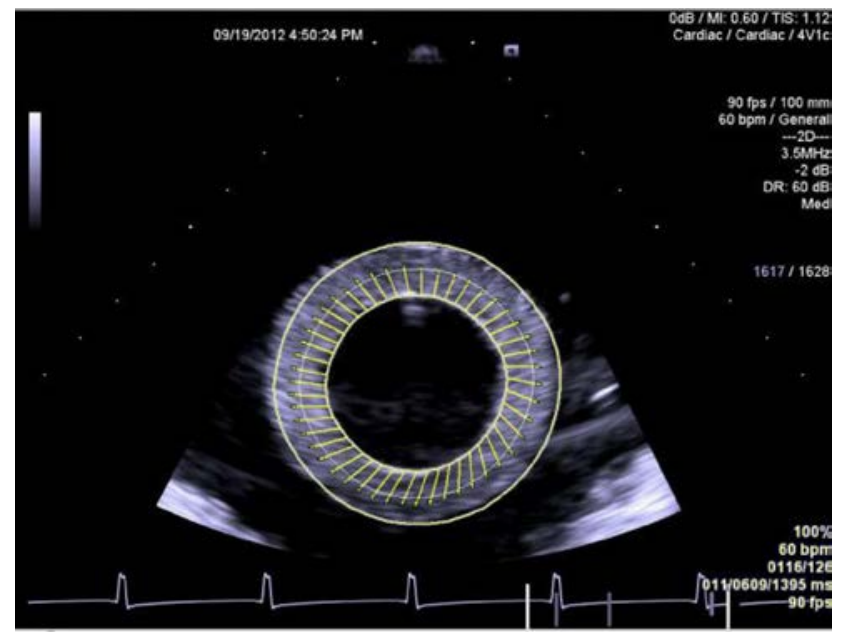

b)

c)

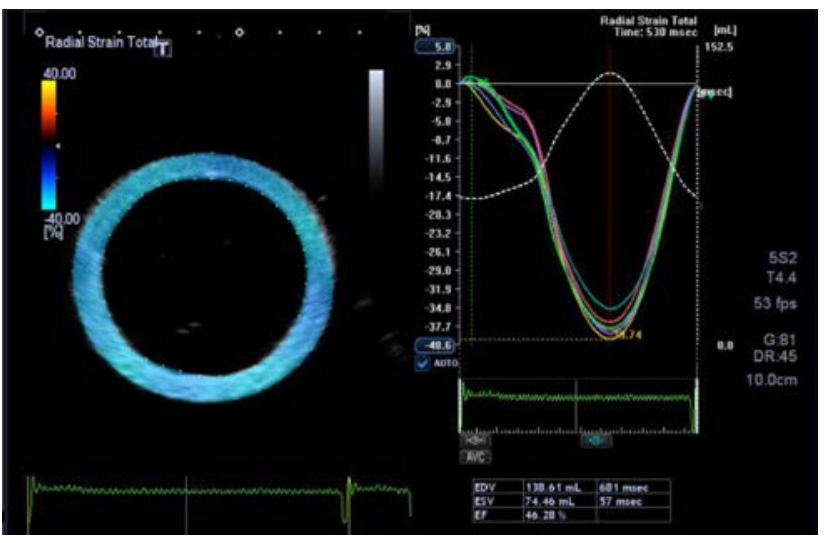

Fig. 17. Echocardiographic image of the model of the proper (healthy) left ventricle: a) two-dimensional imaging in the VVI technique proposed by Acuson, b) two- dimensional imaging in the STE technique proposed by Toshiba, c) two-dimensional deformation (imaging) in the STE technique equal for all the segments (Artida, Toshiba).

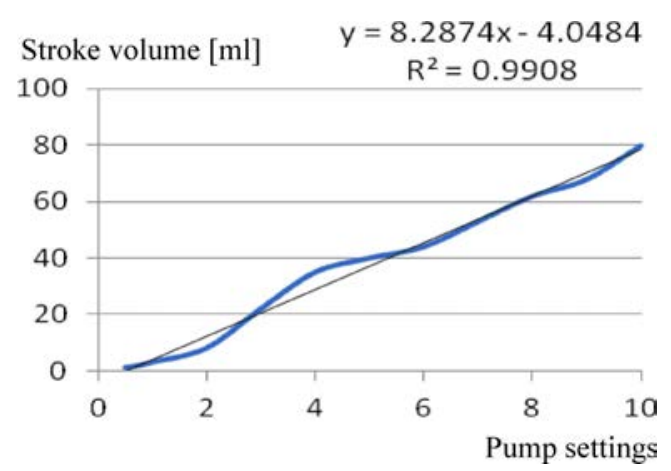

Fig. 18. Diagram presenting the linear dependence of the volume of pumped out water on its dynamics. $y$ - stroke volume; $\mathrm{x}$ - pump settings).

\section{Discussion}

The problem has been solved based on the knowledge falling within the scope of theories of generation of ultrasonic fields, propagation of beams in biologi- 
cal centers, detection and acquisition of echoes from the examined left ventricular wall. Process of scattering presented in the previous paragraph indicates that small areas of muscle with its unique speckle pattern may be defined as "model" for acoustic markers (AM). Image of this model in the cardiac muscle may be observed with commercially available software during the cardiac cycle. During the AM tracking we define the area (complex speckle, group of speckles) that can be observed by applying appropriate algorithm. This algorithm "recognizes" the most similar systems of speckles while going through the adjacent, previously given image cells during the cardiac cycle. For example, the algorithm examines the regions with the smallest difference in the total number of pixels (light) or uses the correlation methods. This technique is independent of the ultrasound beam angle, as it is based on the displacement of speckles, the distribution of which is connected with the characteristics of cardiac muscle walls and not with the characteristics of the ultrasonic beam (with set beam values) as it is in Doppler's technique.

It should be noted that in case presented in Fig. 13 the complex concentration of scatterers is about ten times smaller than average physiological value for real tissue. Nevertheless, the image is complex enough and undergoes significant changes during the deformation. This sets very high requirements for the aforementioned tracking algorithms.

The developed computer modeling enables to perform many experiments unconstrained by the ethics; to analyze the details of the phenomena; to test the diagnostic methods and to calibrate the norms and pathologies for the left ventricle muscle. We used solvers of nonlinear and linear acoustic $4 \mathrm{D}$ field in which the ultrasonic beam propagates and at the same time undergoes scattering in the medium. Software was written in FORTRAN language and MATHCAD programming environment which also enables - based on the speckle imaging - to search for new phenomena of diagnostic significance.

The presented idea of modeling generated by the transducers (including multi-component) of the acoustic field and simulation of the characteristics of transmitter-receivers is significant because of the possibility of increasing the level of accuracy and objectivity of the diagnostics of changes in norm and pathology. Thus, it enables the sooner and better therapy with relatively low financial resources (PICANO, 2005; OTERo et al., 2010). Moreover, it is of great scientific and educational value (MAECKEN et al., 2011; EvANGELISTA et al., 2008).

\section{Ultrasound model of the left ventricle}

The material POLY (VINYL ALCOHOL), 99+\% HYDROLYZED complied with the requirements of the experiment (Fig. 14). The material used to create model of the was suitable for processing. This material is durable and does not require additional process of venting before each experiment.

Conducted model of the LV test, with various volumes of pumped water, as well as with various loads, revealed the compliance of the obtained strain and strain rate results, both for the ultrasonic beam angle of $90^{\circ}$ and $65^{\circ}$. The test was conducted for HR: 40, 80, 100 and 120 cycles per minute and imitated different states of left ventricle, from bradycardia to tachycardia. Usage of Vivitro Inc. pump enables to analyse further physiological and pathological states of LV model because of the linear character of the dependence of pump dynamics on its stroke volume (Fig. 18).

LESNIAK-Plewinska et al., 2010, in their model of left ventricle also pointed out the possibility of measuring left ventricle strains during in vitro experiment. However, they focused on testing the physiological LV strains by imitating more ellipsoidal shape of the ventricle and on the dependence of left ventricle functions on its connection with right ventricle. The author's model of the left ventricle imitates the ring with precisely defined shape and physical parameters.

In the future it may be used to conduct further, deeper analysis of cardiac wall strains on the border between the healthy muscle and pathological area, regardless of the region affected by the pathological process. LV models that have been used to date constitute unique instruments for testing the algorithms of setting parameters connected with the local elasticity of cardiac wall, which may allow to use this method to teach medical practitioners during their specialization and to detect various pathological changes that lead to the segmential stiffening of the left ventricular walls.

\section{Conclusions}

The proposed numerical model and model of the left ventricle are the realization of a fully controlled diagnostic environment, allowing for the testing of algorithms that follow and analyze scattering of speckles, as well as improving the existing algorithms or supporting the construction of new ones.

The mathematical model of the left ventricle, in the described environment of ultrasonographic visualization, allows for the testing of algorithms that follow and analyze scattering of acoustic markers as well as improve the existing algorithms or support the construction of new ones.

Presented ultrasonographic model may serve to analyze left ventricle wall strains in physiological as well as pathological conditions.

\section{Acknowledgment}

The authors greatly acknowledge the financial support (in part) of the National Science Centre (Grant Number N N518 292340). 


\section{References}

1. Bijnens B., D'Hooge J., Sutherland G., HerRegods M.C., Nuyts J., Suetens P., VAn DE WERF F. (1999), Robustness of integrated backscatter for myocardial tissue characterization, Ultrasound in Medicine \& Biology, 25, 95-103.

2. Blotekaer K.I., Ingebrigtsen K.A., Skeie H. (1973), A method for analyzing waves in structures consisting of metal strips on dispersive media, IEEE Trans. Electron Devices, 20, 12, 1133-1138.

3. Bohs L.N., Trahey G.E. (1991), A novel method for angle independent ultrasonic imaging of blood flow and tissue motion, IEEE Transactions on Bio-medical Engineering, 38, 280-286.

4. Evangelista A., Flachskampf F., Lancellotti P., Badano L., Aguilar R., Monaghan M., Zamorano J., Nihoyannopoulos P. (2008), European Association of Echocardiography. European Association of Echocardiography recommendations for standardization of performance, digital storage and reporting of echocardiographic studies, Eur. J. Echocardiogr., $\mathbf{9}, 4,438-448$.

5. Feigenbaum H., Amstrong W.F., Ryan T. (2005), Feigenbaum's echocardiography, Lippincott Williams \& Wilkins.

6. IsAAz K. (2000), What are we actually measuring by Doppler tissue imaging?, Journal of the American College of Cardiology, 36, 897-899.

7. Korinek J., Wang J., Sengupta P.P., MiyazaKi C., KJaergaard J., McMahon E., AbraHAM T.P., BELOHLAVEK M. (2005), Two-dimensional strain - a Doppler-independent ultrasound method for quantitation of regional deformation: Validation in vitro and in vivo, Journal of the American Society of Echocardiography: official publication of the American Society of Echocardiography, 18, 1247-1253.

8. Langeland S., D'Hooge J., Torp H., Bijnens B., Suetens P. (2003), Comparison of time-domain displacement estimators for two-dimensional rf tracking, Ultrasound in Medicine \& Biology, 29, 1177-1186.

9. Leitman M., Lysyansky P., Sidenko S., Shir V., Peleg E., Binenbaum M., Kaluski E., Krakover R., VERED Z. (2004), Two-dimensional strain - a novel software for real-time quantitative echocardiographic assessment of myocardial function, Journal of the American Society of Echocardiography: official publication of the American Society of Echocardiography, 17, 1021-1029.

10. Lesniak-Plewinska B., Cygan S., Kaluzynski K., D'Hooge J., Zmigrodzki J., Kowalik E., KordyBACH M., KOWALSKI M. (2010), A dual-chamber, thick-walled cardiac phantom for use in cardiac motion and deformation imaging by ultrasound, Ultrasound in Medicine \& Biology, 36, 1145-1156.

11. Lloyd-Jones D., Adams R.J., Brown T.M., Carnethon M., Dai S., De Simone G., Ferguson T.B., Ford E., Furie K., Gillespie C., Go A., Greenlund K., HaAse N., Hailpern S., Ho P.M.,
Howard V., Kissela B., Kittner S., Lackland D., Lisabeth L., Marelli A., McDermott M.M., Meigs J., Mozaffarian D., Mussolino M., NiChol G., Roger V.L., Rosamond W., Sacco R., Sorlie P., Roger V.L., Thom T., WAsserthielSmoller S., Wong N.D., Wylie-Rosett J. (2010), Heart disease and stroke statistics - 2010 update: A report from the American Heart Association, Circulation, 12, 1, e46-e215.

12. Maecken T., Zinke H., Zenz M., Grau T. (2011), How should anesthesiologists perform ultrasound examinations? Diagnostic use of ultrasound in emergency and intensive care and medicine, Anaesthesist., 60, 3, 203-213.

13. Mor-Avi V., Lang R.M., Badano L.P., Belohlavek M., Cardim N.M., Derumeaux G., Galderisi M., Marwick T., Nagueh S.F., Sengupta P.P., Sicari R., Smiseth O.A., Smulevitz B., Takeuchi M., Thomas J.D., Vannan M., Voigt J.U., Zamorano J.L. (2011), Current and evolving echocardiographic techniques for the quantitative evaluation of cardiac mechanics: ASE/EAE consensus statement on methodology and indications endorsed by the Japanese Society of Echocardiography, European Journal of Echocardiography: the journal of the Working Group on Echocardiography of the European Society of Cardiology, 12, 167-205.

14. Olszewski R., Timperley J., Szmigielski C., Monaghan M., Nihoyannopoulos P., Senior R., BECHER H. (2007), The clinical applications of contrast echocardiography, European Jjournal of Echocardiography: the journal of the Working Group on Echocardiography of the European Society of Cardiology, 8, S13-S23.

15. Otero H.J., Rybicki F.J., Greenberg D., Mitsouras D., Mendoza J.A., Neumann P.J. (2010), Cost-effective diagnostic cardiovascular imaging: when does it provide good value for the money? Int. J. Cardiovasc. Imaging, 26, 6, 605-12.

16. Picano E. (2005), Economic and biological costs of cardiac imaging, Cardiovasc. Ultrasound, May 25, 313.

17. Van de Veire N.R., De Sutter J., Bax J.J., RoeLANDT J.R. (2008), Technological advances in tissue Doppler imaging echocardiography, Heart, 94, 10651074.

18. Wagner R.F., Smith S.W., SAndrik J.M., LoPEZ H. (1983), Statistics of speckle in ultrasound Bscan, IEEE Trans. Sonics and Ultrasonics, 30, 156-163.

19. Wojcik J., Kujawska T., Nowicki A., Lewin P.A. (2008), Fast prediction of pulsed nonlinear acoustic fields from clinically relevant sources using timeaveraged wave envelope approach: Comparison of numerical simulations and experimental results, Ultrasonics, 48, 707-715.

20. Wojcik J., Nowicki A., Lewin P.A., BloomField P.E., Kujawska T., Filipczynski L. (2006), Wave envelopes method for description of nonlinear acoustic wave propagation, Ultrasonics, 44, 310-329. 